Supplementary information

\title{
Reliably Probing the Conductance of a Molecule in a Cavity via van der Waals Contacts
}

Mingzhu Huang, ${ }^{\dagger}{ }^{\S} \nabla$ Mingjun Sun, ${ }^{\dagger}, \nabla$ Xiang Yu, ${ }^{\dagger}$ Suhang He, ${ }^{\#}$ Simin Liu, ${ }^{\ddagger}$ Werner M.

Nau, ${ }^{\#}$ Yunchuan $\mathrm{Li},{ }^{\dagger}$ Tao Wu,, Yun Wang, ${ }^{\perp}$ Shuai Chang,,${ }^{* \dagger}$ Jin He ${ }^{*}, \S$

The State Key Laboratory of Refractories and Metallurgy, the Institute of Advanced Materials and Nanotechnology, College of Materials and Metallurgy, Wuhan University of Science and Technology, Wuhan, Hubei, 430081, China.

School of Chemistry and Chemical Engineering, Wuhan University of Science and Technology, Wuhan, Hubei, 430081, China.

${ }^{\S}$ Department of Physics, Biomolecular Science Institute, Florida International University, Miami, Florida 33199, USA.

"Department of Life Sciences and Chemistry, Jacobs University Bremen, Bremen 28759, Germany.

"School of Information Science and Technology, ShanghaiTech University, Shanghai, 201210, China.

${ }^{\perp}$ Center for Clean Environment and Energy, School of Environment \& Science, Griffith University, Queensland, 4222, Australia.

Corresponding author: schang23@wust.edu.cn, jinhe@ fiu.edu 


\section{Table of Content}

S1. Chemicals and Electrodes

S2. The home-built STM-BJ setup and single-molecule conductance measurement

S3. Different types of G-D traces obtained from CB[7]-FC junctions in STM-BJ measurements

S4. The conductance histograms of $\mathrm{CB}[7]-\mathrm{FC}$ at two gain settings of the current amplifier

S5. The conductance histograms of single-molecule junctions containing guest molecules only

S6. The conductance histograms of other host-guest complexes

S7. I-V measurements of CB[7]-FC junctions at different gap distances

S8. Details of theoretical calculations

S9. The optimized Au/ferrocene/Au structures with different gap distances

S10. The calculated transmission spectra and total energies

S11. Interaction between FC molecule and Au electrodes

S12. The optimized molecular geometries

S13. The calculated charge density distributions of $\mathrm{CB}[7]-\mathrm{FC}$ with both $\mathrm{FC}$ orientations

S14. The calculated I-V curves

S15. Calculated molecular projected self-consistent Hamiltonian (MPSH) states

S16. Structural model of Au/FC/Au junction for quantum transport calculations

S17. Summaries of $G_{H}$ and $G_{L}$ values obtained from junctions containing different host-guest complexes

S18. Supramolecular studies of cucurbit[7]uril and guests 


\section{S1. Chemicals and Electrodes}

Cucurbit[7]uril (CB[7]) was synthesized following the published procedure ${ }^{1-3}$, the content of the sample was $83 \%$ as determined by quantitative NMR. Aminoferrocene (AFC) was purchased from TCL AMERICA (Portland, USA), Ferrocene (FC) was purchased from Alfa Aesar (USA). 4-Phenylpyridine was purchased from Toyoko Chemical Industry Co., Lt. Aminobutane was purchased from Sigma-Aldrich. 1, 2, 4-tricholorobenzene (TCB), Amantadine were purchased from Sigma-Aldrich (Saint Louis, USA). All chemicals were used without further purification. All aqueous solutions were prepared in deionized (DI) water ( 18 M ohm, Purelab system, ELGA/Siemens). CB[7] solution was prepared by dissolving $\mathrm{CB}[7]$ powders into DI water to a final concentration of $1 \mathrm{mM}$. The hostguest complex solutions were prepared by mixing the host (CB[7]) and guest molecule at a ratio of $1: 1$ in water, followed by a treatment of sonication for $\sim 30$ minutes $^{4}$. For CB[7]-FC, different ratios at 1:0.5, 1:1 and 1:1.5 stoichiometry in DI water were also prepared to conduct the measurements plotted in Figure 2C. FC is less soluble than other guest molecules, so the sonication took more than $1 \mathrm{~h}^{5}$. Final concentration of the host-guest complexes are $1 \mathrm{mM}$.

The gold tips were made from $0.2 \mathrm{~mm}$ diameter gold wires (Electron Microscopy Sciences, $99.99 \%$ pure) through electrochemical etching ${ }^{6,7}$. Before usage, they were cleaned with piranha solution $\left(1: 3 \mathrm{H}_{2} \mathrm{O}_{2}: \mathrm{H}_{2} \mathrm{SO}_{4}\right.$. Cautions, extremely corrosive!), DI water and pure ethanol, and dried in nitrogen gas flow. Gold substrates were prepared by sequentially evaporating $5 \mathrm{~nm} \mathrm{Cr}$ and $100 \mathrm{~nm}$ gold on silicon substrates with an electron beam evaporator. Before chemical modification, the gold substrates were cleaned with oxygen plasma for 10 minutes and hydrogen flame annealing for $1 \mathrm{~min}$. Subsequently, they were immersed in the molecule solutions for overnight. Before usage, they were rinsed with DI water and dried in nitrogen gas flow.

\section{S2. The home-built STM-BJ setup and single-molecule conductance measurement}

The experiments are conducted with a home-built single axis STM. The position of the STM probe is controlled with a piezo actuator (S-303.0L PI, Germany) driven by an analog output (NI PXle-4463 card 24 bits) in the range of $-10 \mathrm{~V}$ to $10 \mathrm{~V}$. An analog input (NI PXle-4309 18 bits at $2 \mathrm{MS} / \mathrm{s} / \mathrm{ch}$ ) is used to record the current, which is amplified by a variable range current-voltage converter (DLPCA-200, Femto, Germany). We typically use three gains for the current measurements. By converting the current values to conductance in the unit of $G_{0}\left(G_{0}\right.$ $\left.=2 \mathrm{e}^{2} / \mathrm{h}=77,481 \mathrm{nS}\right)$ using a constant bias $(0.1 \mathrm{~V}$, if not mentioned otherwise), the typical dynamic ranges for the $10^{6}, 10^{7}$ and $10^{9}$ gains of the current amplifier are $1 \times 10^{-5} \mathrm{G}_{0}-1 \times 10^{2} \mathrm{G}_{0}, 1 \times 10^{-6} \mathrm{G}_{0}-1 \times 10^{-1} \mathrm{G}_{0}$, and $5 \times 10^{-7}$ $\mathrm{G}_{0}-1 \times 10^{-3} \mathrm{G}_{0}$, respectively.

The STM-BJ experiments are controlled by a custom Labview program. The tip is repeatedly approached to and then retracted from the substrate at a constant bias $(0.1 \mathrm{~V}$, if not mentioned otherwise). For each cycle, the gold tip approaches to the gold substrate with PID (proportional-integral-derivative control) control until the tip contacts the substrate to reach a conductance around $10 \mathrm{G}_{0}$. Then, the tip withdraws a distance of $35 \mathrm{~nm}$ at a speed of $50 \mathrm{~nm} / \mathrm{s}$. The typical total time for each cycle is about $2 \mathrm{~s}$. The recorded G-D curves are analyzed by a custom labview program. To avoid large background noise, the traces with conductance $\mathrm{G}$ decays slowly and stretches more than $2 \mathrm{~nm}$ distances are discarded, which is less than $2 \%$ of all the data. All the results in the main text are performed in TCB solution. We have also performed the STM-BJ measurement of CB[7] in DI water using partially insulated tip. As shown in Figure S1, no obvious conductance peak can be identified. However, conductance peak of $\mathrm{CB}[7]$ can be identified when the experiments are conducted in TCB solution. In a previous report ${ }^{8}$, no conductance peak was observed for the STM-BJ measurement of CB[8] in water. Therefore, the polarity of the solvent impacts the interaction between gold and carbonyl groups.

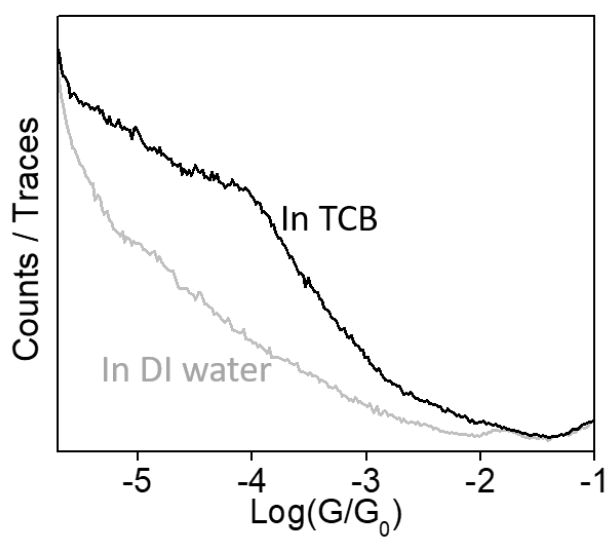

Figure S1. The normalized conductance histograms of $\mathrm{CB}[7]$ measured in DI water (gray) and TCB solution (black). Each histogram is built from more than 5000 G-D traces. 


\section{S3. Different types of G-D traces obtained from CB[7]-FC junctions in STM-BJ measurements}

In the G-D traces of CB[7]-FC junction used to construct the conductance histogram, there are about four types as shown in Figure S2a. The G-D traces showing both $G_{H}$ and $G_{L}$ steps are close to $50 \%$ of all the G-D traces.
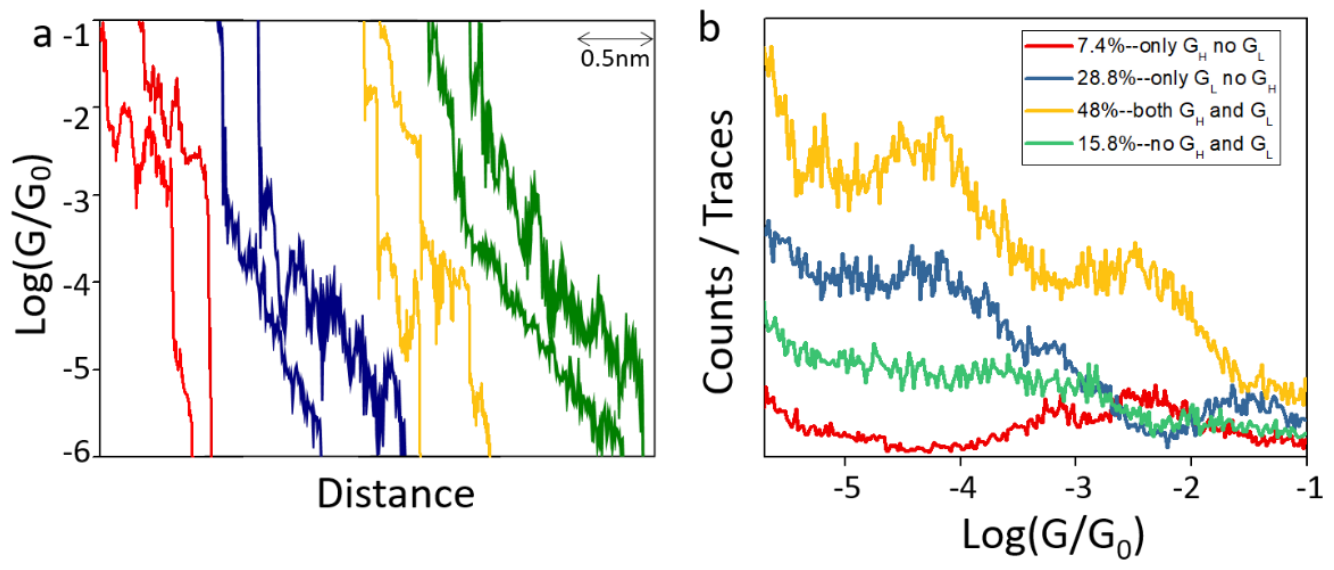

Figure S2. Different types of G-D traces of CB[7]-FC. (a) Different types of G-D traces: 1) red color traces, only obvious steps near $\mathrm{G}_{\mathrm{H}}$ (about $10^{-2.5} \mathrm{G}_{\mathrm{O}}$ ); 2) navy color traces, only obvious steps near $\mathrm{G}_{\mathrm{L}}$ (about $10^{-4}$ to $10^{-}$ ${ }^{5} \mathrm{G}_{0}$ ); 3) yellow color traces, obvious steps appear near both $\mathrm{G}_{H}$ and $\mathrm{G}_{\llcorner}$; 4) olive color traces, noisy traces with current spikes/steps all over the conductance range. (b) The normalized conductance histograms constructed from four types of G-D traces. The ratio of different type of traces are also given.

\section{S4. The conductance histograms of $\mathrm{CB}[7]-\mathrm{FC}$ at two gain settings of the current amplifier}

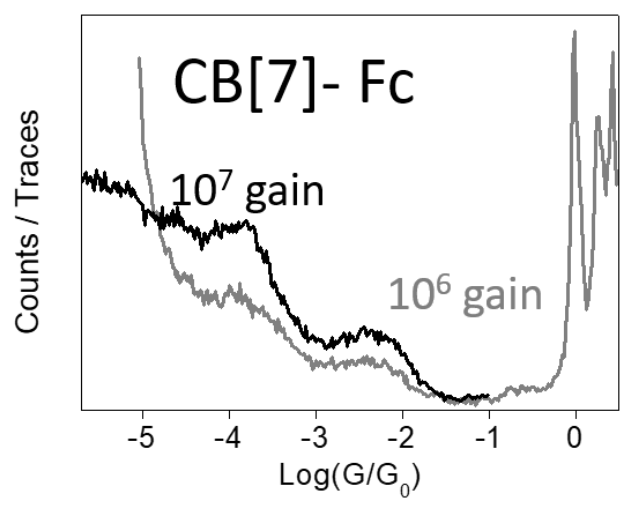

Figure S3. The normalized conductance histograms of CB[7]-FC obtained by the current amplifier at $1 \times 10^{6}$ gain (gray color trace) and $1 \times 10^{7}$ gain (black color trace). 
S5. The conductance histograms of single-molecule junctions containing guest molecules only
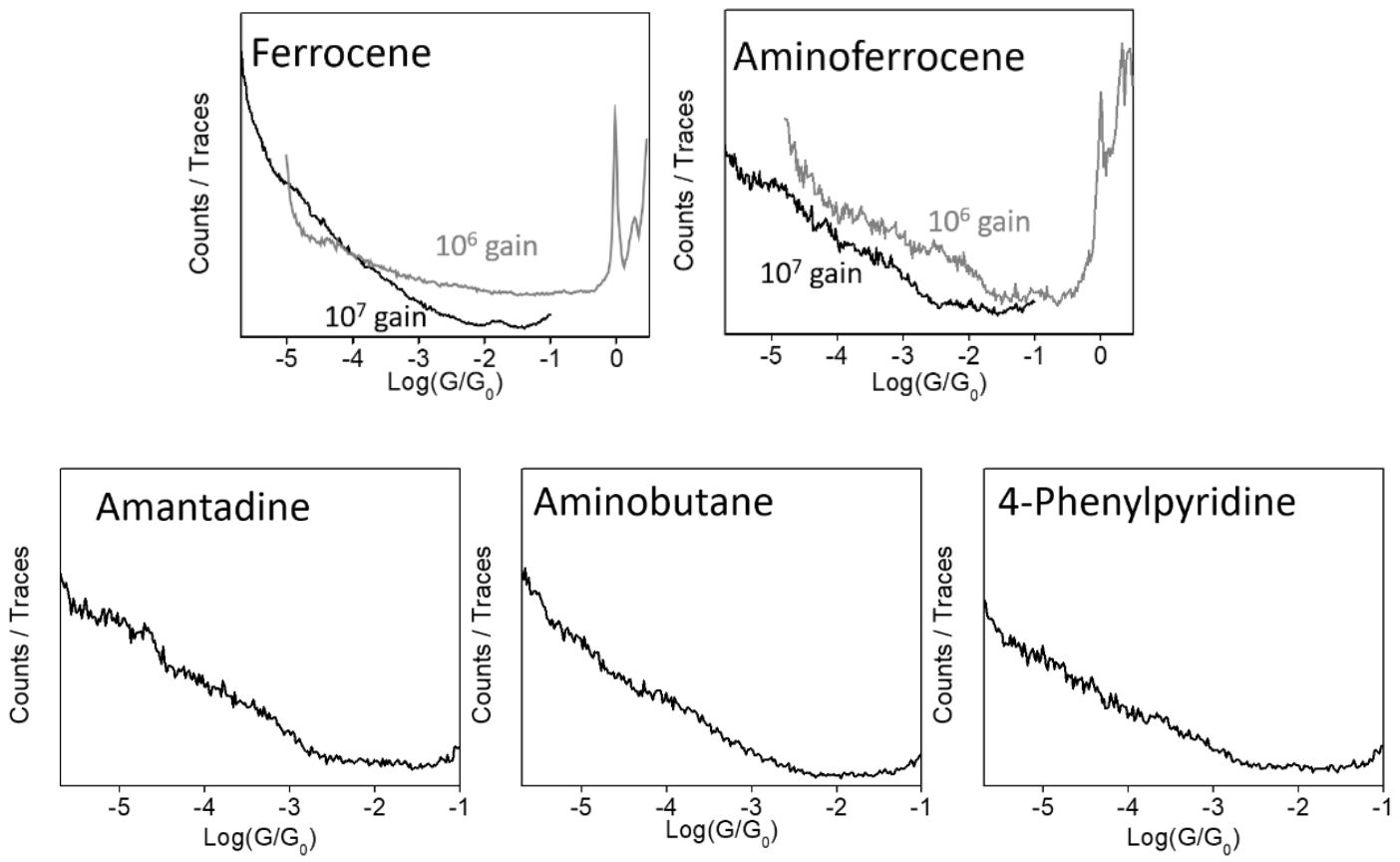

Figure S4. The normalized conductance histograms (counts/trace) of different guest molecules. For ferrocene and aminoferrocene molecules (top panel), two conductance histograms by two current amplifier gains are shown for each molecule to cover about 6 orders conductance range. Before measurements, the gold substrates are immersed in molecule solution (DI water containing $1 \mathrm{mM}$ molecule) for $24 \mathrm{~h}$. The measurements are conducted in TCB solution.

\section{S6. The conductance histograms of different host-guest complexes}

Figure S5 a to d show the conductance histograms for four different host-guest complexes, which are constructed from combined conductance decay traces without any data selection. The typical conductance decay traces are listed to the right of each histogram and can be generally separated into four types (see descriptions for different types of curve in Figure S2). The onset ratio for different types is listed on top of each corresponding trace, which is obtained by analyzing at least three separate experiments. For all four host-guest systems, both low conductance $\left(\mathrm{G}_{\mathrm{L}}\right)$ plateaus and high conductance $\left(\mathrm{G}_{\mathrm{H}}\right)$ plateaus are observed in the typical decay traces, showing that both $\mathrm{CB}[7]$ and the guest molecule are detected and probed during the measurements, as explained for the case of $\mathrm{CB}[7]-\mathrm{FC}$ in the main text. However, the onset frequency of $\mathrm{G}_{H}$ plateaus for $\mathrm{CB}[7]$-aminobutane and $\mathrm{CB}$ [7]-4-phenylpyridine is much lower than the other complexed systems. This trend can be better visualized by comparing the overall $G_{H}$ ratio (sum of the ratio for " $G_{H}$ only" and " $G_{H}+G_{L}$ ") for different systems, which represents the probability for sensing the guest molecules during the measurements. As shown in Figure $\mathrm{S6}$, the overall $\mathrm{G}_{H}$ plateaus (red dots) are observed up to $60 \%$ of all traces for $\mathrm{CB}[7]$ ferrocene, $\mathrm{CB}[7]$-aminoferrocene and $\mathrm{CB}[7]$-amantadine. However, for $\mathrm{CB}[7]$-aminobutane and $\mathrm{CB}[7]-4$ phenylpyridine, $G_{H}$ occurrence dropped to $20 \%$ (circled in Figure S6). The sharp decay of the $G_{H}$ ratio might be attributed to the different binding affinity between guest molecules and CB[7]. As shown in Table 1 in the manuscript, ferrocene, aminoferrocene and amantadine have much higher binding affinity toward $\mathrm{CB}[7]$ than aminobutane and 4-phenylpyridine, so greater chance would take place for the first three guests to stay inside $\mathrm{CB}[7]$ cavity (rather than diffusing out) during the break junction measurements, thus generating a higher $\mathrm{G}_{\mathrm{H}}$ ratio. 


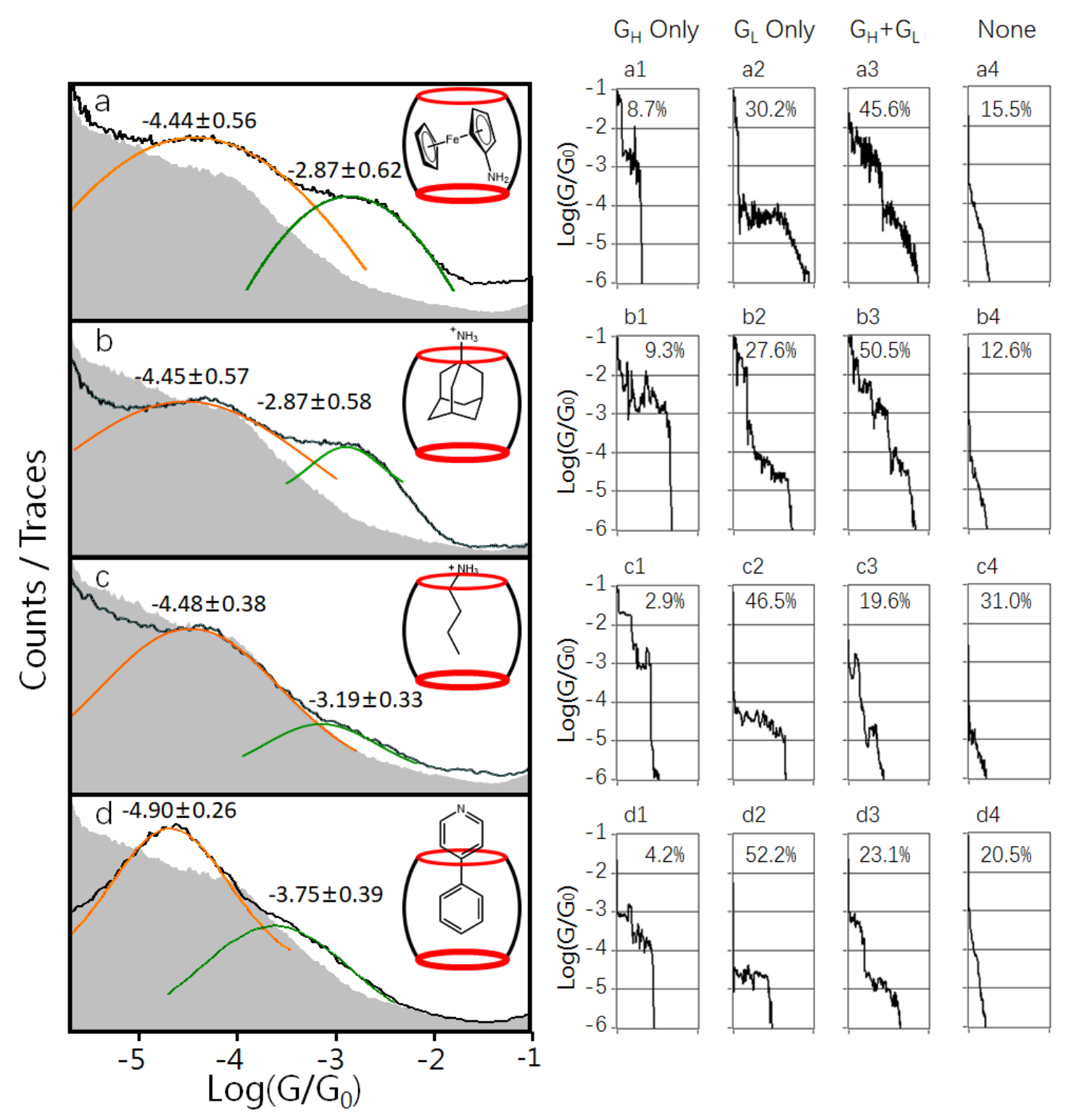

Figure S5. Conductance histograms for junctions containing (a) CB[7]-aminoferrocene, (b) CB[7]-amantadine , (c) CB[7]-aminobutane , (d) CB[7]-4-phenylpyridine . The gray area shows the conductance histogram of CB[7] from Figure $2 \mathrm{~b}$. The black line shows the conductance histogram of different host guest complex. The orange and olive solid lines are Gaussian fits to the $G_{L}$ and $G_{H}$ conductance peaks. Typical conductance decay traces for different host-guest complexes are displayed to the right of each histogram. Four types of traces are clear for each complexed system with the onset frequency listed on top of each corresponding trace. The concentration ratio of [guest]/[host] was 1 in all the molecule solution. All the STM-BJ measurements were conducted in TCB solution with amplifier gain at $1 \times 10^{7}$. 


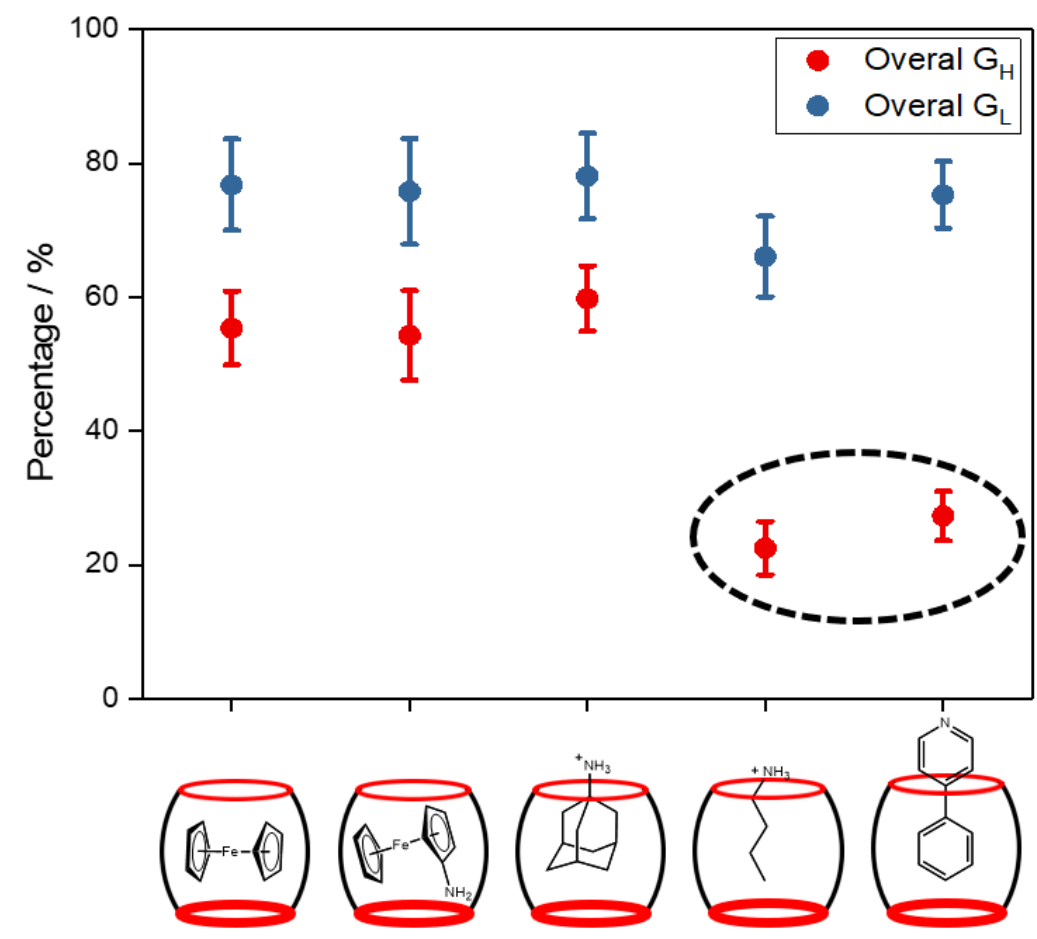

Figure S6. Overall $G_{H}$ (blue dots) and $G_{L}$ (red dots) frequency observed in all decay traces for different hostguest complexes. The $\mathrm{G}_{H}$ frequency points noted with a black circle show that the high conductance features for $\mathrm{CB}[7]-$ aminobutane and $\mathrm{CB}[7]-4-$ phenylpyridine are much lower than the other three complexed systems.

\section{S7. I-V measurements of CB[7]-FC junctions at different gap distances}

The I-V curves (Figure 2d) of the molecular junctions are obtained by the following procedure. First, the STM tip is approached to the molecule modified gold substrate to reach a preset current value $\mathrm{I}_{\mathrm{b}}$ at a constant bias $(\mathrm{V}=$ $0.1 \mathrm{~V}$ ) with PID control. The current set point determines the gap distance. While the tip is on the substrate, the PID parameters are reduced to very small values $(P=0.1, I=0.1, D=0)$ or set to zero (open-loop). When the molecular junction is formed, the baseline current $I_{b}$ is noticeably more stable. We then perform I-V measurements in the bias range $-1 \mathrm{~V}-+1 \mathrm{~V}$ with the servo off. After the bias sweep, the current normally returns to the original value $I_{b}$ (see Figure S5), suggesting the molecular junction is not changed or broken during the bias sweep. When the current is stable, we repeat the I-V measurement. We only choose the I-V curves when the baseline current returns to the initial value after the bias sweep, which suggests that the molecular junctions are stable during the course of the IV measurement. It should be noted that the acquired I-V curves may be from junctions containing one or a few molecules. 

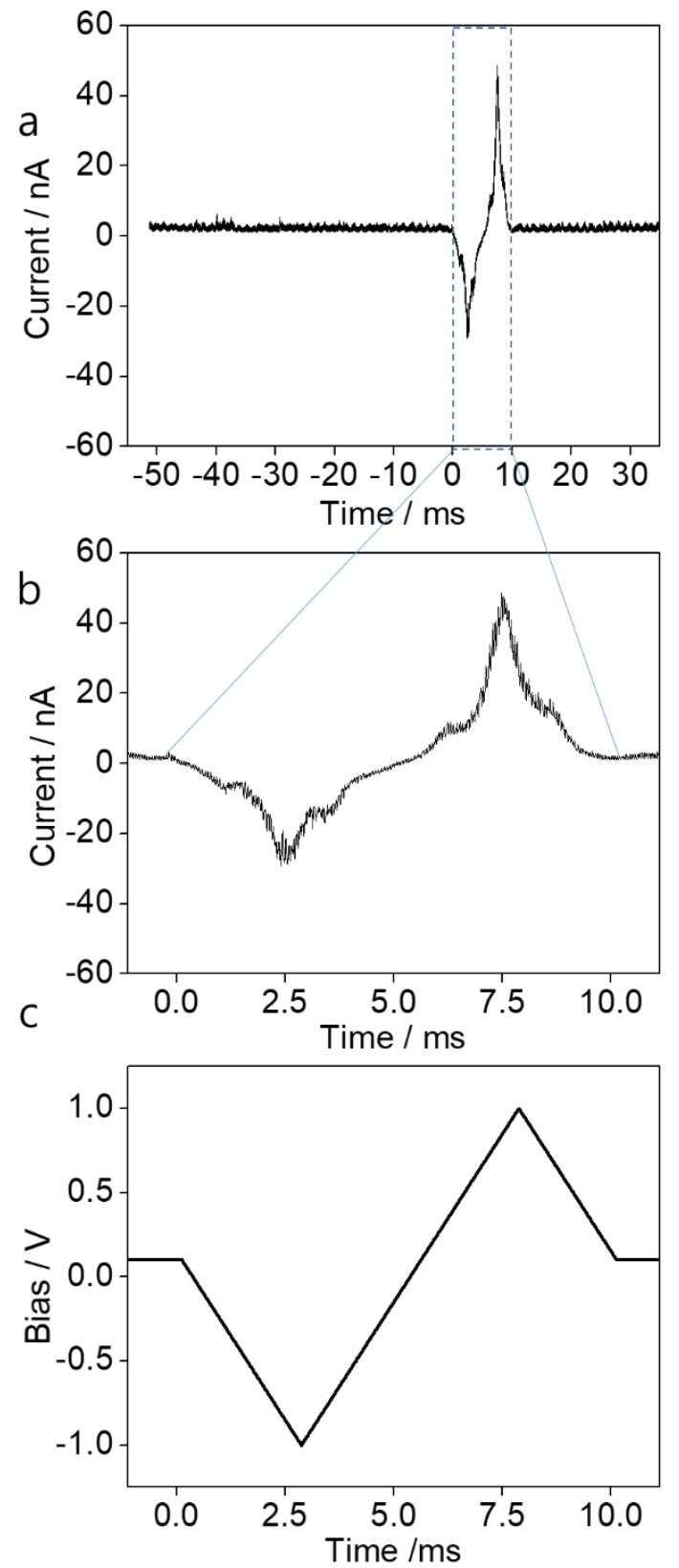

Figure S7. I-V measurements. A. The typical I-t trace during the I-V measurements. B. The zoom-in of the I-t curve in the region enclosed by the blue dashed line square, showing the current change during the I-V measurement. C. The bias sweep during the I-V measurement.

The I-V curves are sensitive to the gap distance between tip and substrate, which is controlled in experiment by the baseline current $I_{b}$, or baseline conductance $G_{b}\left(G_{b}=I_{b} / V, V\right.$ is the applied bias). The tip is positioned at different gap distances from the substrate surface based on the baseline conductance. The gap distance $d$ is estimated based on the baseline conductance $G_{b}$ using the following equation: $G_{b}=G_{0}{ }^{*} \exp (-\beta d)$, where $G_{0}$ is the quantum of conductance, $\beta$ is the measured decay constant of the TCB solvent $\left(6.48 \mathrm{~nm}^{-1}\right)$. 

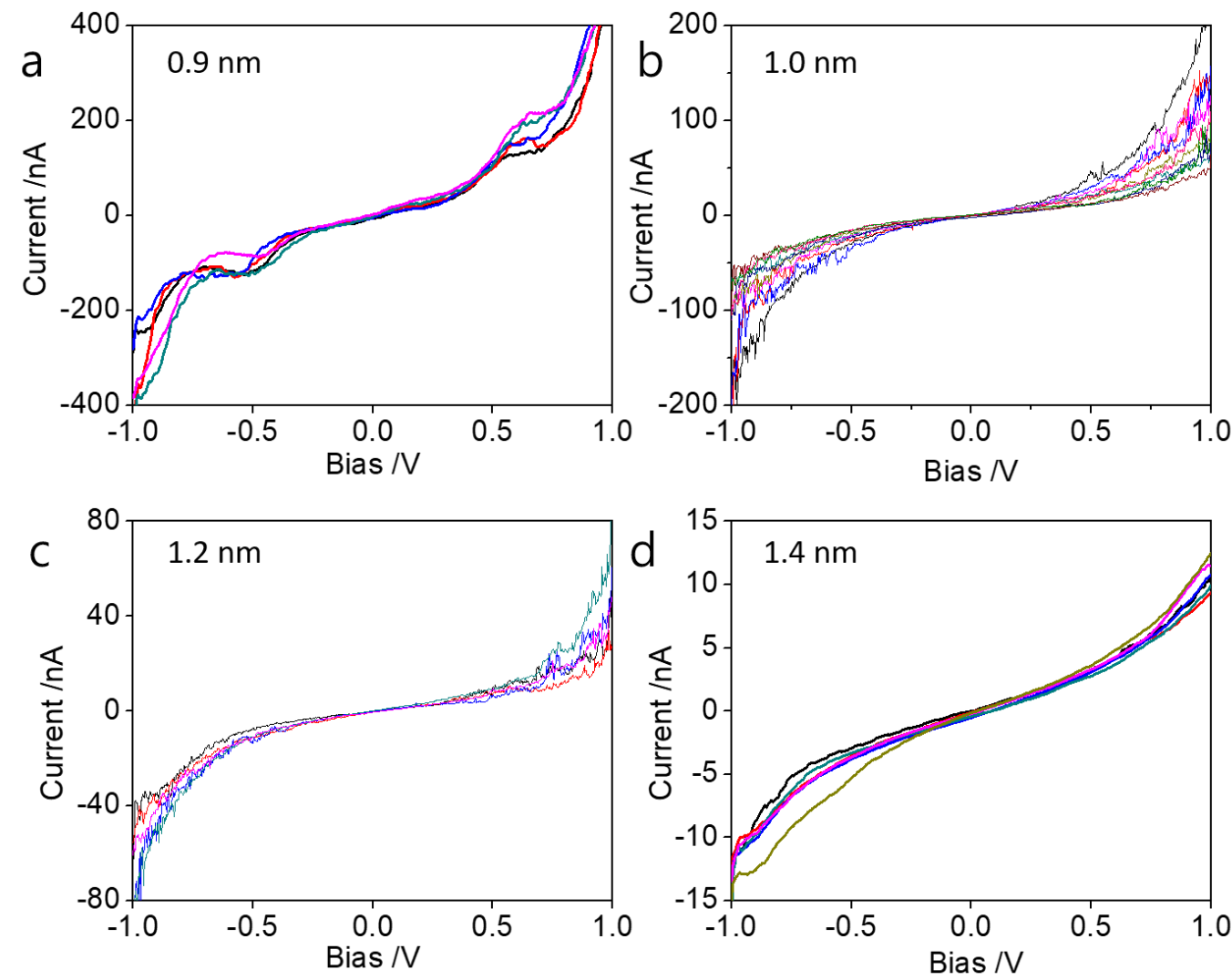

Figure S8. I-V curves measured at different gap distances $\left(G_{b}=10^{-2.53} G_{0}\right.$ for $0.9 \mathrm{~nm}, G_{b}=10^{-2.81} G_{0}$ for $1 \mathrm{~nm}$, $\mathrm{G}_{\mathrm{b}}=10^{-3.37} \mathrm{G}_{0}$ for $1.2 \mathrm{~nm}, \mathrm{G}_{b}=10^{-3.94} \mathrm{G}_{0}$ for $1.4 \mathrm{~nm}$ ). The nonlinear behavior is obvious when the gap distance is about $0.9 \mathrm{~nm}$.

\section{S8. Details of theoretical calculations}

The optimization of the scattering region and the electronic conductance calculations are performed by using $\mathrm{Au} / \mathrm{FC} / \mathrm{Au}$ junctions with the NEGF-DFT formalism at the Perdew-Burke-Ernzerhof (PBE-GGA) level implemented in ATK program. The double- $\zeta$ polarized (DZP) basis set is used for all atoms except gold, and the single- $\zeta$ polarized (SZP) basis set is used for gold atoms. A mesh cut-off of 150 Ryb, electron temperature of $300 \mathrm{~K}$, density matrix convergence of $10^{-4}$, and residual forces on each atom smaller than $0.05 \mathrm{eV} / \AA$ are adopted. The k-point sampling grid is taken to be $3 \times 3 \times 100$, three k-points in the irreducible of the Brillouin zone for the $x$ and $y$-directions, and $100 \mathrm{k}$-points for the $z$-direction (the transport direction of the junction). The electrodes are simulated using $\mathrm{Au}$ (111) surfaces, in which the right electrode is composed of five-layer $4 \times 4 \mathrm{Au}(111)$ and the left electrode is composed of six-layer $4 \times 4 \mathrm{Au}$ (111). Meanwhile, the outermost $\mathrm{Au}$ atom of the left electrode occupies hollow sites on Au (111) surface for Au tip. Thereinto, the bottom three gold layers are fixed, and other atoms are relaxed and optimized. The transmission spectra are calculated using a large set of $5 \times 5 \mathrm{k}$-points based on the optimized structures, and the I-V curve and the molecular projected self-consistent Hamiltonian (MPSH) are also simulated. To compare the corresponding energies, the molecular geometries of CB[7]-FC complexes with two structural states are optimized using the same method as the Au/FC/Au junctions by the ATK program. To explore the interaction energies between $\mathrm{Au}$ electrodes and $\mathrm{FC}$ molecule, the $\mathrm{Au}_{20}$ cluster was used to represent the electrode, and the interaction energies as a function of the Au-FC distance are calculated using the B3LYP-D3 function. Here the LanL2DZ basis set is used for the Fe atom, and the basis set of $6-31 \mathrm{G}(\mathrm{d}, \mathrm{p})$ is used for all other atoms. Moreover, the BSSE corrected binding energies are also considered. The binding energy $\left(\mathrm{E}_{\mathrm{b}}\right)$ is defined by $\mathrm{E}_{\mathrm{b}}=\mathrm{E}_{\mathrm{Au}_{20}-\mathrm{FC}}-\mathrm{E}_{\mathrm{Au}_{20}}-\mathrm{E}_{\mathrm{FC}}+\Delta \mathrm{E}_{B S S E}, \mathrm{E}_{\mathrm{Au}_{20}}, \mathrm{E}_{\mathrm{FC}}, \mathrm{E}_{\mathrm{Au}_{20}-\mathrm{FC}}$ are the energy of $\mathrm{Au}_{20}$ cluster, $\mathrm{FC}$, and $\mathrm{Au}_{20}-\mathrm{FC}$ complex, respectively. We also performed a theoretical and numerical analysis of the different counterpoise correction (CP) schemes potentially applicable to correct for the basis set superposition error (BSSE).

\section{S9. The optimized $\mathrm{Au} / \mathrm{FC} / \mathrm{Au}$ structures with different gap distances}

Figure $\mathrm{S} 8$ displays the optimized structures at different gap distances. 


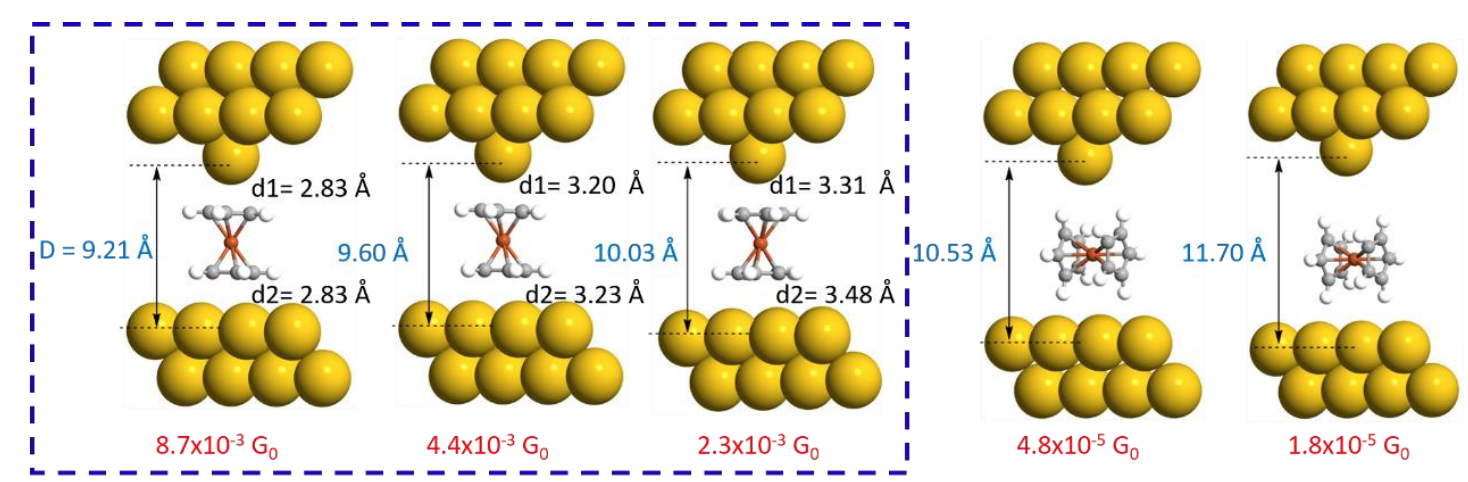

Figure S9. The optimized scattering region of the $\mathrm{Au} / \mathrm{FC} / \mathrm{Au}$ junctions with the different distances between the electrodes. $\mathrm{D}$ is the distance between gold substrate and the gold tip, d1 is the distance between Au tip and the center point of the upper $\mathrm{C}_{\mathrm{P}}$ ring of FC molecule, and $\mathrm{d} 2$ is the distance between the gold substrate and the center point of the lower $\mathrm{C}_{\mathrm{p}}$ ring.

\section{S10. The calculated transmission spectra and total energies}

As shown in the transmission spectra in Figure S9, the HOMO of FC is closer to the Fermi level of gold electrode than the LUMO of FC. The $\varepsilon_{\mathrm{H}}\left(=\mathrm{E}_{\mathrm{F}}-\mathrm{E}_{\mathrm{HOMO}}\right)$ is between $0.2 \mathrm{~V}-0.8 \mathrm{~V}$ depending on the gap distance $\mathrm{D}$. The $\varepsilon_{\mathrm{L}}$ $\left(=\mathrm{E}_{\mathrm{LUMO}}-\mathrm{E}_{\mathrm{F}}\right)$ is bigger than $2 \mathrm{~V}$.

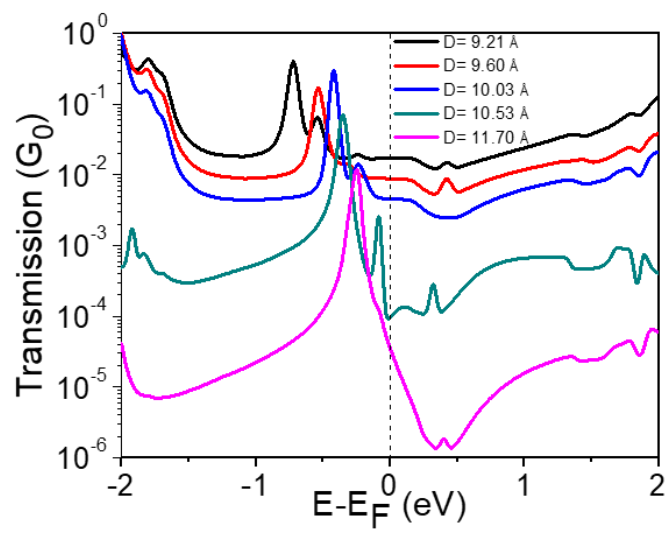

Figure S10. The transmission spectra of the $\mathrm{Au} / \mathrm{FC} / \mathrm{Au}$ junctions at zero-bias with different gap distances (as shown in Figure S8) between the electrodes, and D is the distance between gold substrate and the gold tip.

Figure S10 shows the calculated energy of the optimized geometries of $\mathrm{Au} / \mathrm{FC} / \mathrm{Au}$ junction at different gap distance $D$ for vertical-FC (Evertical) and horizontal-FC ( $\left.E_{\text {Horizontal }}\right)$ systems. Given that the height of $C B[7]$ is $9.1 \AA$, we show the calculated results from $\mathrm{D}=9.21 \AA$. In Figure $3 \mathrm{~b}, \Delta \mathrm{E}$ is set as the total energy difference between the vertical and horizontal states of $F C, \Delta E=\left(E_{\text {vertical }}+E_{d}\right)-E_{\text {Horizontal }}\left(E_{d}\right.$ is the energy difference between the horizontal and vertical states of FC in the $\mathrm{CB}[7]$ cavity in the absence of gold electrodes, see S12). 


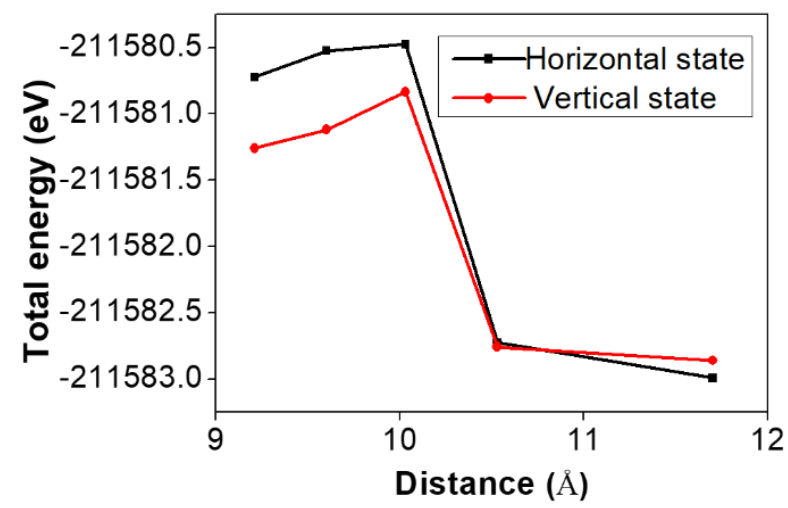

Figure S11. The calculated total energies for $\mathrm{Au} / \mathrm{FC} / \mathrm{Au}$ junctions with different gap distances (as shown in Figure S8) between the electrodes.

\section{S11. Interaction between FC molecule and Au electrodes}

To explore the interaction energies between Au electrodes and FC molecule, the Au20 cluster is used to represent the electrode, and the interaction energies as a function of the Au-FC distance are calculated using the B3LYP-D3 function. The details of parameter settings are shown in supplementary information S8

Corresponding to the increase of the gap distance $D$ from $9.21 \AA$ to $10.03 \AA$, the distance d1 between the gold tip and the center of the CP ring of FC (at the vertical orientation) is changed from 2.8 to $3.3 \AA$ (see the optimized $\mathrm{Au} / \mathrm{FC} / \mathrm{Au}$ geometry in Figure S8). In this distance range, the interaction energy between gold probe and FC stays in the range of -5 to $-10 \mathrm{kcal} / \mathrm{mol}$, as in Figure $\mathrm{S} 11 \mathrm{a}$. Similarly, the interaction energy between the flat gold substrate and FC at the vertical orientation is also calculated during the same changing process of $D$, showing an energy in the range of -10 to $-15 \mathrm{kcal} / \mathrm{mol}$. The values and distance-dependence of these energies are indicative of vdW interactions between FC and both Au electrodes. The circled points in the following plots are shown as Figure $3 \mathrm{~d}$ in the main text.

a

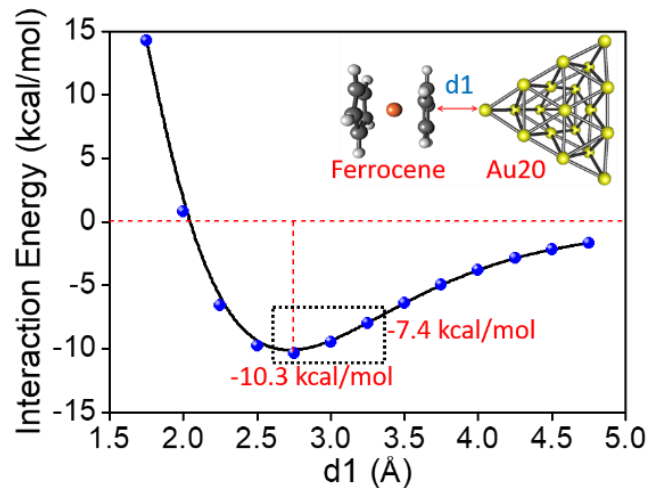

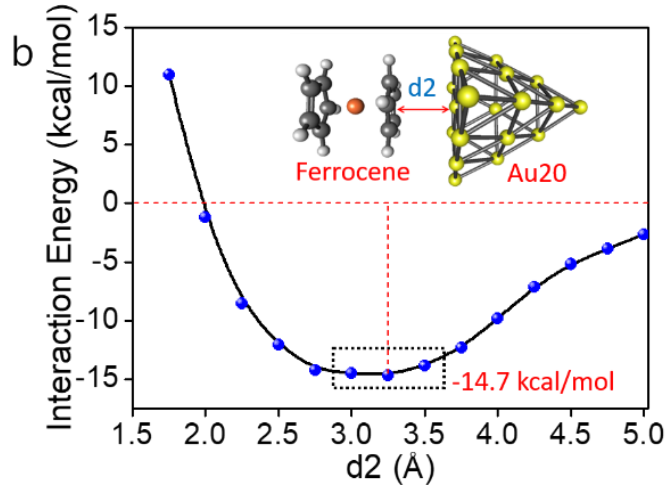

Figure S12. The changes of Au-FC interaction energies with the electrode gap increase at different contact geometry.

\section{S12. The optimized molecular geometries}

Figure S12 shows the optimized complexes of CB[7]-FC with different symmetries (D5d and D5h). More stable singlet state of the FC molecule, shown in Table S1, is used for all structural calculations. In our calculations, there is a total energy difference of $E_{d}=4.31 \mathrm{kcal} / \mathrm{mol}$ between the horizontal and vertical states of $F C$ in the $\mathrm{CB}[7]$ cavity, as shown in Figure c. FC is more stable with the horizontal orientation inside the $\mathrm{CB}$ [7] cavity, which is consistent with the previous calculations. ${ }^{9,10}$ 
a

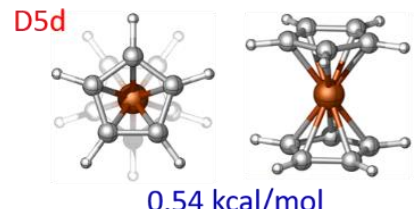

$0.54 \mathrm{kcal} / \mathrm{mol}$

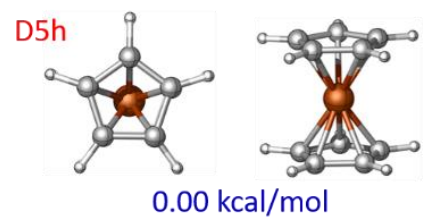

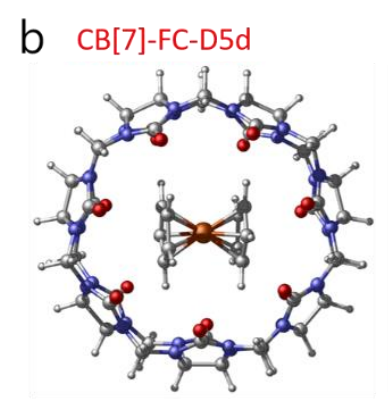

$0.00 \mathrm{kcal} / \mathrm{mol}$

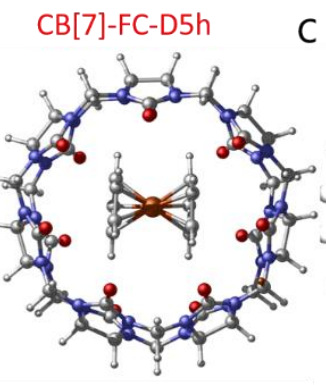

$0.04 \mathrm{kcal} / \mathrm{mol}$
C Horizontal state

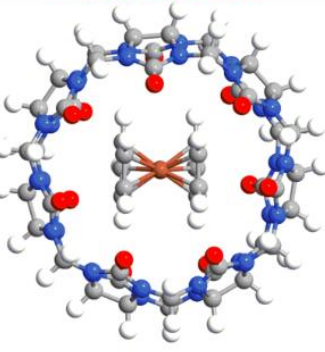

$0.00 \mathrm{kcal} / \mathrm{mol}$
Vertical state

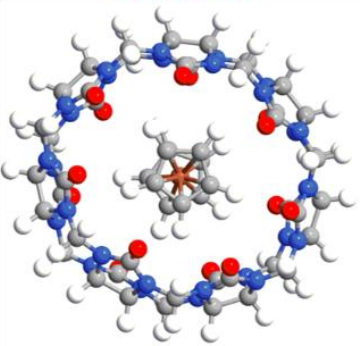

$+4.31 \mathrm{kcal} / \mathrm{mol}$

Figure S13. The optimized molecular geometries. (a) The optimized structures of FC with different symmetries (D5d and D5h). (b) The optimized complexes of CB[7]-FC with different symmetries (D5d and D5h). (c) Energy difference between two complexed configurations in the absence of gold electrodes.

Table S1. The energy difference of the FC molecule with different spin multiplicities.

\begin{tabular}{c|c|c}
\hline Spin multiplicity & $\mathrm{E}_{\text {zero-point }}(\mathrm{kcal} / \mathrm{mol})$ & Energy difference $(\mathrm{kcal} / \mathrm{mol})$ \\
\hline 1 & -1650.731995 & 0.0 \\
\hline 3 & -1650.707302 & 15.5 \\
\hline 5 & -1650.712305 & 12.4 \\
\hline
\end{tabular}

\section{S13. The calculated charge density distributions of $\mathrm{CB}[7]-\mathrm{FC}$ with both $\mathrm{FC}$ orientations}

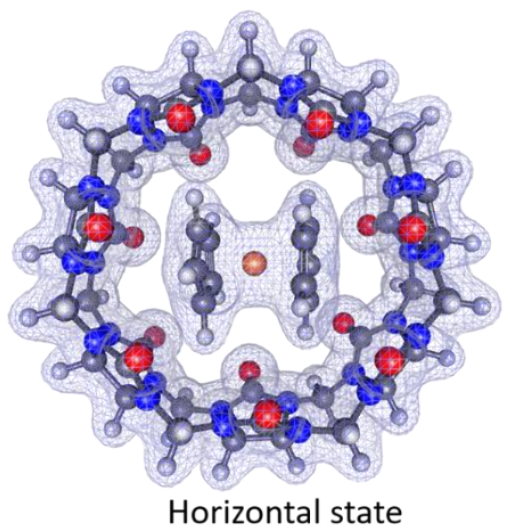

Horizontal state

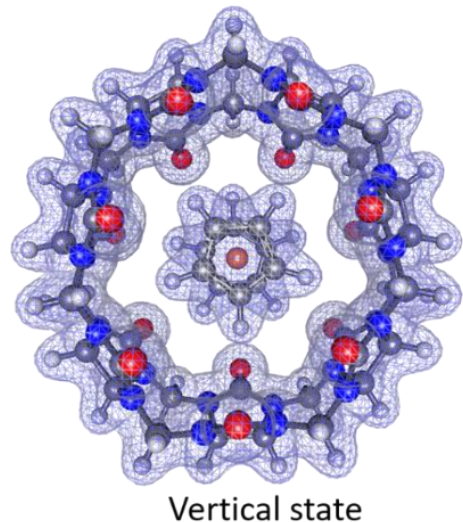

Vertical state

Figure S14. The calculated electron densities of the CB[7]-FC complexes with different orientations of FC inside the $\mathrm{CB}[7]$ (left, Horizontal state; right, vertical state). The isovalue is 0.25 a.u., and the oxygen, nitrogen and carbon atoms are red, blue and gray color, respectively. 


\section{S14. The calculated I-V curves}

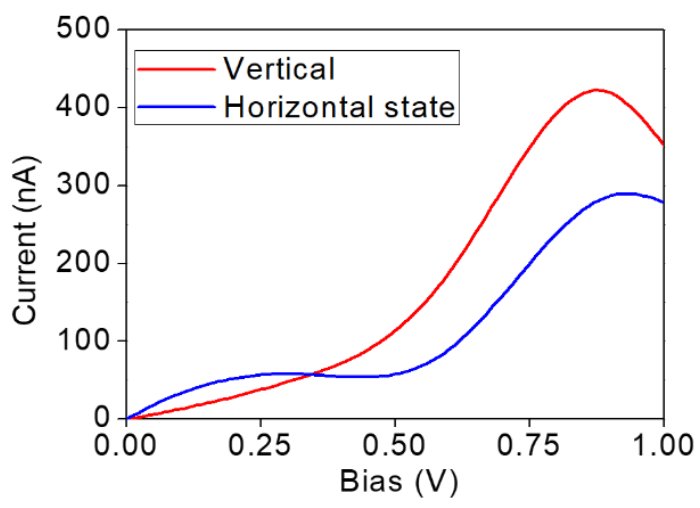

Figure S15. The NEGF-DFT method computed I-V curves of the Au/FC/Au junctions with two FC orientations (red, vertical state; blue, Horizontal state) between two gold electrodes.

\section{S15. Calculated molecular projected self-consistent Hamiltonian (MPSH) states}

The orbital composition analysis shows that the HOMO is contributed by the Au electrodes and the whole FC molecule, while the LUMO is only localized in the left Au electrode.

a

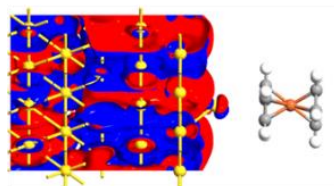

LUMO+1
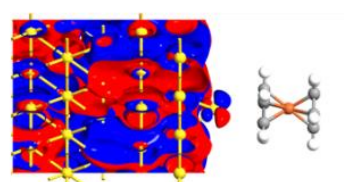

LUMO

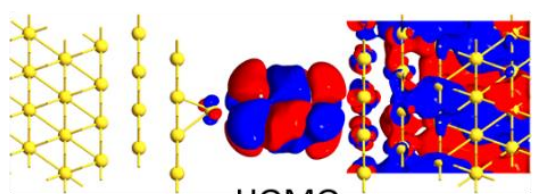

HOMO

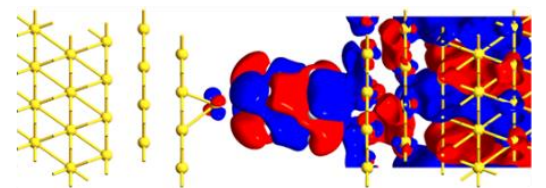

HOMO-1

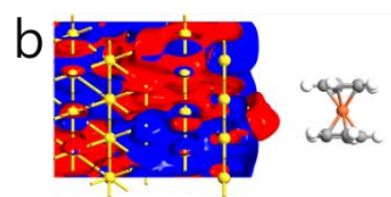

LUMO+1

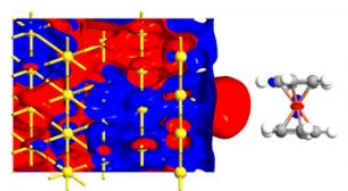

LUMO
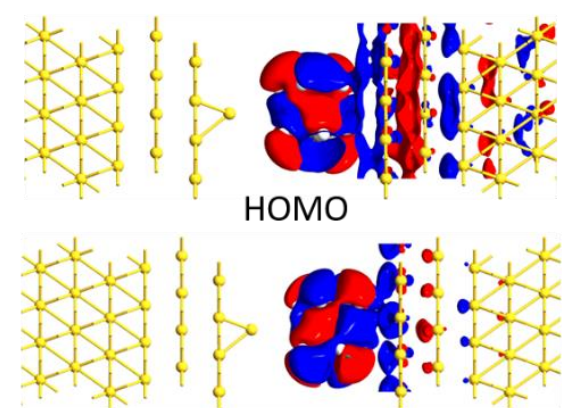

HOMO-1

Figure S16. Molecular projected self-consistent Hamiltonian (MPSH) states of the Au/FC/Au junctions (a, the vertical state; $b$, the horizontal state) contributing to the peaks in the transmission spectra.

\section{S16. Structural model of $\mathrm{Au} / \mathrm{FC} / \mathrm{Au}$ junction for quantum transport calculations}

Considering that a few gold atoms are often pulled out to become adatoms during the STM-BJ measurements, we introduce a low-coordinated gold atom (hollow site) at the top electrode. To reduce the computation cost, $\mathrm{CB}[7]$ molecule is not included in the model of Au/FC/Au junction. Instead, the effect of $\mathrm{CB}[7]$ is taken into account by simply adding the energy difference $E_{d}$ (see S12) between two FC orientations in the CB[7] cavity into the energy of $\mathrm{Au} / \mathrm{FC} / \mathrm{Au}$ junction model with $\mathrm{FC}$ at the vertical orientation. Based on the calculated electron density distribution of the $\mathrm{CB}[7]-\mathrm{FC}$ complexes in Figure S13, the electronic couplings between $\mathrm{CB}[7]$ and $\mathrm{FC}$ 
in both orientations are small. Therefore, the $\mathrm{Au} / \mathrm{FC} / \mathrm{Au}$ junction model without $\mathrm{CB}[7]$ should be a reasonable model for the experiments here.

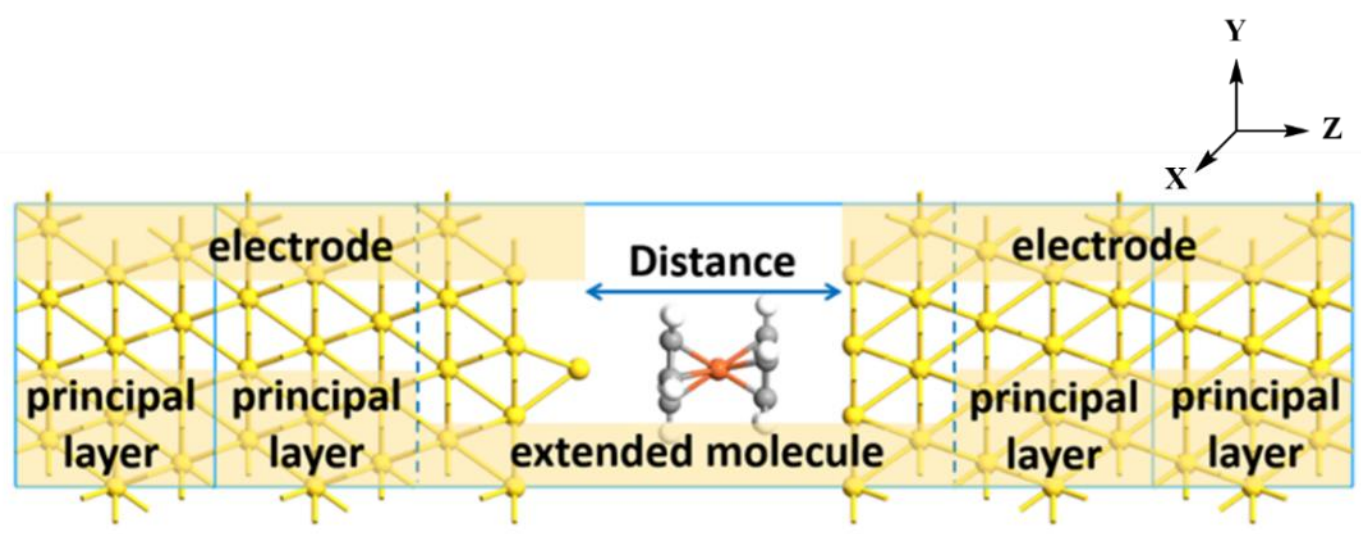

Fig.S17. Exemplary configuration of the Au/FC/Au junction.

\section{S17. Summaries of $G_{H}$ and $G_{L}$ values obtained from junctions containing different host-guest complexes}

Figure S17 show conductance values of junctions containing CB[7]-aminoferrocene, CB[7]-amantadine, CB[7]aminobutane, and $\mathrm{CB}[7]-4-$ phenylpyridine, with their corresponding chemical structures displayed at the bottom of Figure 4. Control experiments of junctions containing only these molecules show conductance histograms with no distinguishable peaks (See Figure S4). The conductance values obtained from CB[7] and CB[7]ferrocene junctions are also listed for comparison. We compared the obtained $G_{H}$ of each host-guest complex to the reported conductance $\left(\mathrm{G}_{\mathrm{ref}}\right)$ of their homologous molecules with two anchor groups. Diamine-ferrocene is homologous to ferrocene and aminoferrocene. The conductance distribution of diamine-ferrocene is reported in a wide range (several conductance peaks span the range of $10^{-3}$ to $10^{-1} \mathrm{G}_{0}$ are reported from a recent MC-BJ measurement ${ }^{11}$. We use the error bar to represent the possible range of the measured conductance values of diamine-ferrocene). The wide conductance range of diamine-ferrocene is explained by the various binding geometries and conformational change of ferrocene during the mechanical stretching of the junction. In contrast, the $\mathrm{G}_{\mathrm{H}}$ of ferrocene or aminoferrocene confined in the $\mathrm{CB}$ [7] cavity yields comparable values but has a narrower conductance distribution. The well-defined conductance peak in our measurements implies that CB[7] cavity could effectively suppress the conformational change of ferrocene between electrodes. Similarly, diaminobutane and dipyridyl (insets in Figure 4) are analogous to aminobutane and 4-phenylpyridine, and their reported conductance values ${ }^{12-14}$ (blue triangle points) are also very close to the measured $\mathrm{G}_{\mathrm{H}}$. It is worth mentioning that amantadine is an important drug molecule ${ }^{15}$ and its single-molecule electrical properties have not been reported to date.

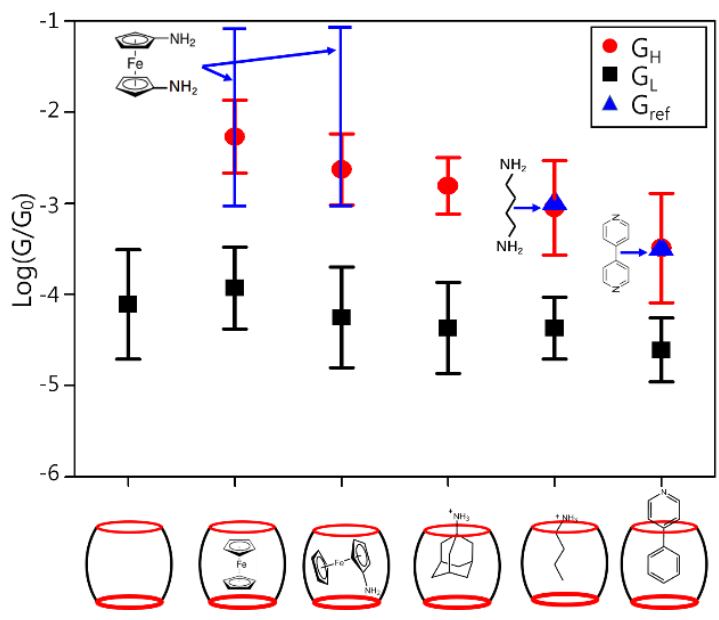

Figure S18. The measured single-molecule conductance values of $\mathrm{CB}[7]$ based host-guest complexes. Both the $G_{L}$ (black solid squares) and $G_{H}$ (red solid spheres) values of different complexes are shown in the plot. The error bar of each value is the standard deviation obtained from the Gaussian fits to the conductance peaks. The reference conductance $\left(G_{\mathrm{ref}}\right)$ of 1,1'-diamine ferrocene is from ref ${ }^{11}$, and 1, 4-diaminebutane is from refs ${ }^{16,17}$, and $4,4^{\prime}$-bipyridine is from ref ${ }^{18}$. 


\section{S18. Supramolecular studies of cucurbit[7]uril and guests}

${ }^{1} \mathrm{H}$ NMR experiments were carried out on a JEOL Oxford AS-400 (400 MHz) spectrometer. Isothermal calorimetric titrations were carried out at $25^{\circ} \mathrm{C}$ in Milli-Q water on a VP-ITC calorimeter from MicroCal, USA

Binding of cucurbit[7]uril and 4-phenylpyridine
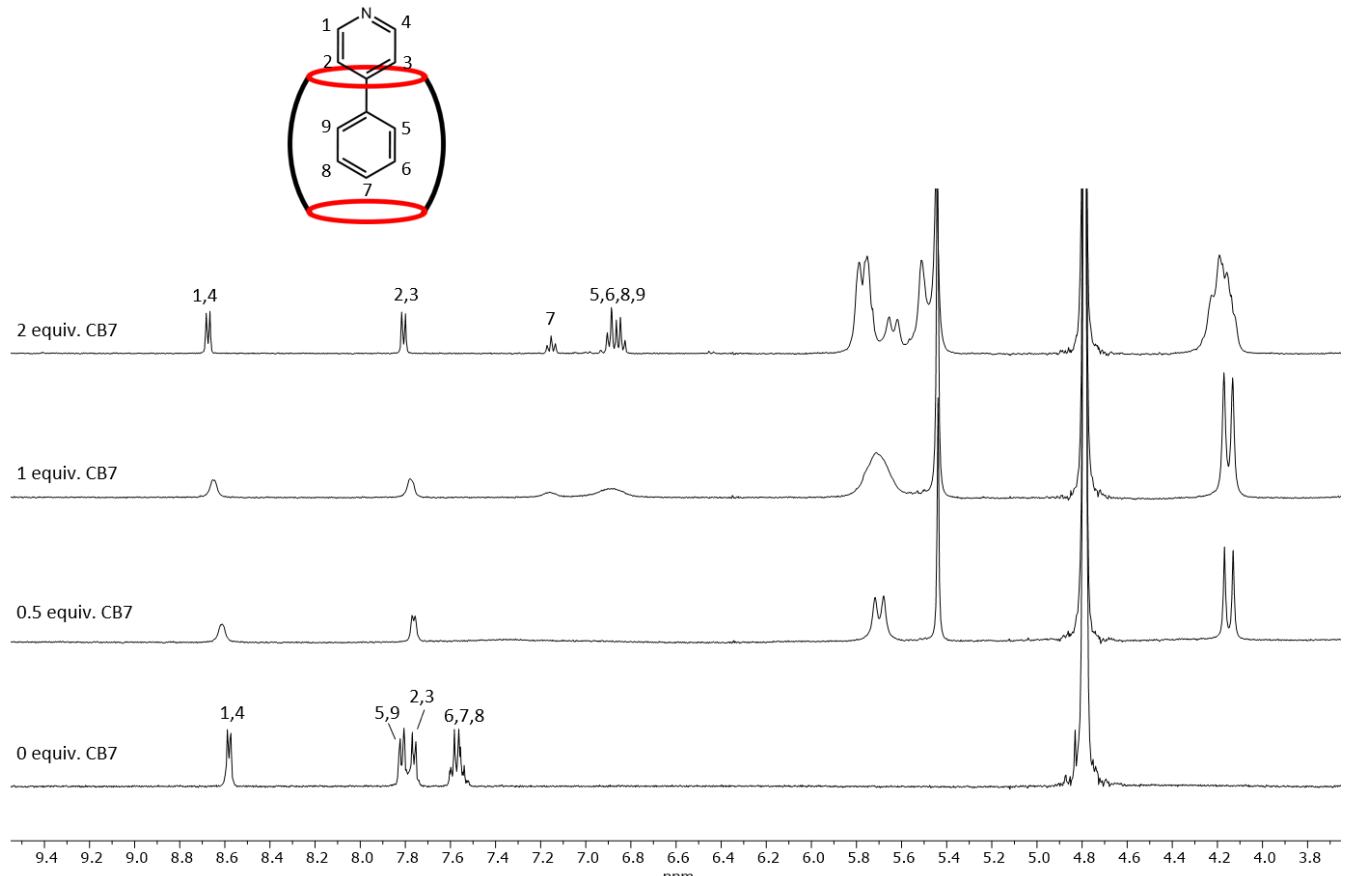

Figure S19. Stacked ${ }^{1} \mathrm{H}$ NMR spectra with proton assignments of 4-phenylpyridine in $\mathrm{D}_{2} \mathrm{O}$ in the presence of 0 equiv, 0.5 equiv, 1 equiv, and 2 equiv CB7 (from bottom to top). Four protons on the pyridine ring $(1,2,3,4)$ were all shifted downfield as the CB7 concentration increased, indicating their position at the carbonyl rim, whereas the other protons all showed apparent up-field shifts, indicating their location in the inner cavity.

Time (min)

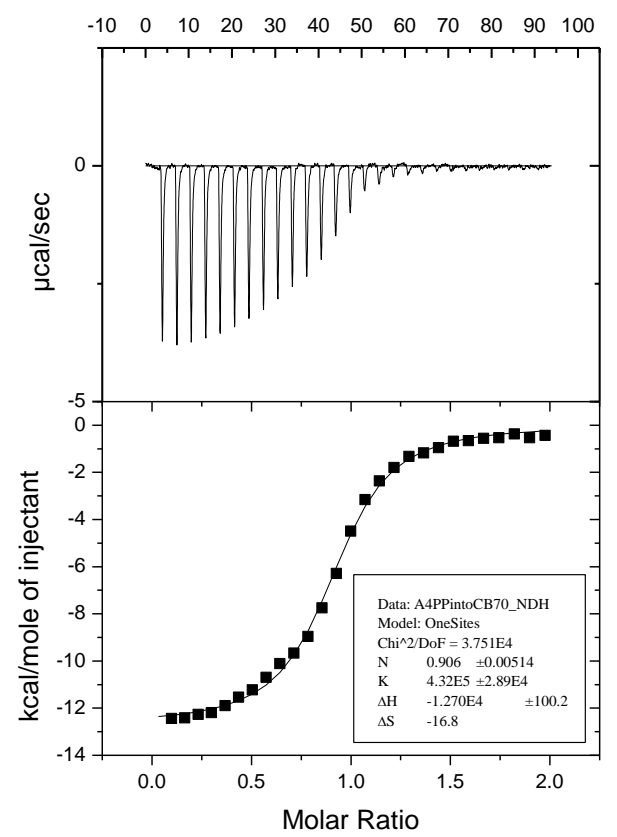

Figure S20. Isothermal calorimetric titration of 4-phenylpyridine and CB7 in water at $298 \mathrm{~K}$ and fitting according to a $1: 1$ stoichiometry. A binding constant of $(4.32 \pm 0.29) \times 10^{5} \mathrm{M}^{-1}$ was determined. 
Binding of cucurbit[7]uril and aminobutane

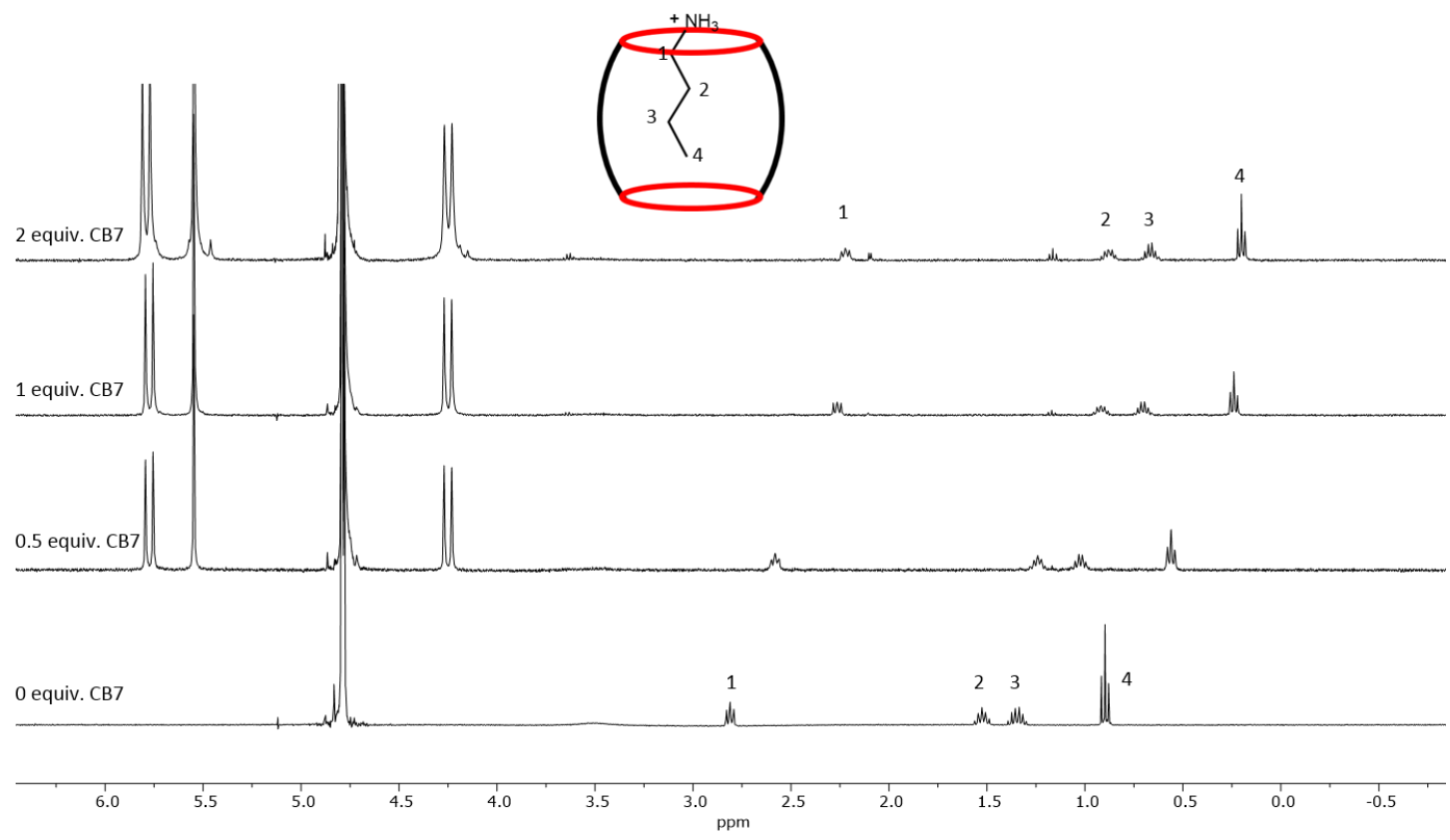

Figure S21. Stacked ${ }^{1} \mathrm{H}$ NMR spectra with proton assignments of aminobutane in $\mathrm{D}_{2} \mathrm{O}$ solution in the presence of 0 equiv, 0.5 equiv, 1 equiv, and 2 equiv CB7 (from bottom to top). The $p K_{a}$ of aminobutane at the testing temperature ( $295 \mathrm{~K}$ for NMR and $298 \mathrm{~K}$ for ITC) is 10.21 (simulated by MarvinSketch 19.8 software), therefore it exists as the protonated form in solution. All alkyl protons (1-4) were shifted upfield upon addition of CB7, indicating they are all located inside the cavity; the protonated ammonium group is attracted to the carbonyl rim by ion-dipole interactions.

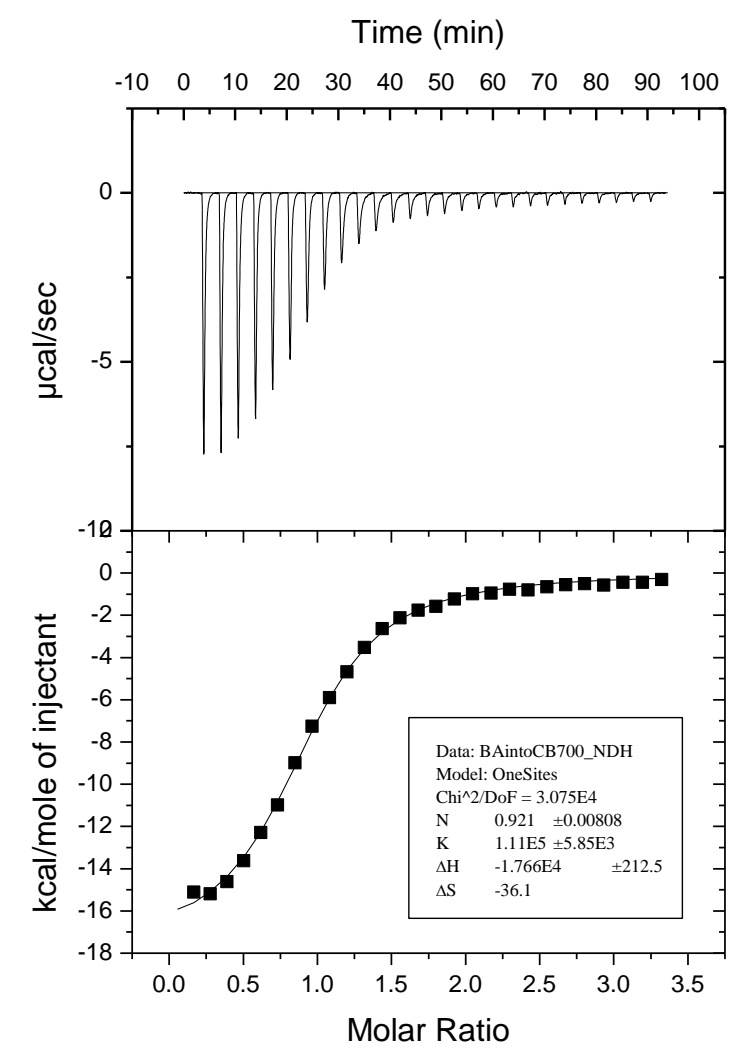

Figure S22. Isothermal calorimetric titration of aminobutane and CB7 in water at $298 \mathrm{~K}$ and fitting according to a $1: 1$ stoichiometry. A binding constant of $(1.11 \pm 0.06) \times 10^{5} \mathrm{M}^{-1}$ was determined. 


\section{References:}

1. Montes - García, V.; Pérez - Juste, J.; Pastoriza - Santos, I.; Liz - Marzán, L. M., Metal nanoparticles and supramolecular macrocycles: a tale of synergy. Chemistry - A European Journal 2014, 20 (35), 10874-10883.

2. Barrow, S. J.; Kasera, S.; Rowland, M. J.; del Barrio, J.; Scherman, O. A., Cucurbituril-Based Molecular Recognition. Chem Rev 2015, 115 (22), 12320-406.

3. Day, A.; Arnold, A. P.; Blanch, R. J.; Snushall, B., Controlling factors in the synthesis of cucurbituril and its homologues. The Journal of organic chemistry 2001, 66 (24), 8094-8100.

4. Sigle, D. O.; Kasera, S.; Herrmann, L. O.; Palma, A.; Denijs, B.; Benz, F.; Mahajan, S.; Baumberg, J. J.; Scherman, O. A., Observing Single Molecules Complexing with Cucurbit[7]uril through Nanogap Surface-Enhanced Raman Spectroscopy. Journal of Physical Chemistry Letters 2016, 7 (4), 704. 5. Xiao, B.; Liang, F.; Liu, S.; Im, J. O.; Li, Y.; Liu, J.; Zhang, B.; Zhou, J.; He, J.; Chang, S., Cucurbituril mediated single molecule detection and identification via recognition tunneling. Nanotechnology 2018, 29 (36).

6. Tuchband, M.; He, J.; Huang, S.; Lindsay, S., Insulated gold scanning tunneling microscopy probes for recognition tunneling in an aqueous environment. Review of Scientific Instruments 2012, 83 (1), 015102.

7. Guo, J.; Pan, J.; Chang, S.; Wang, X.; Kong, N.; Yang, W.; He, J., Monitoring the Dynamic Process of Formation of Plasmonic Molecular Junctions during Single Nanoparticle Collisions. Small 2018, 14 (15), 1704164.

8. Zhang, W.; Gan, S.; Vezzoli, A.; Davidson, R. J.; Milan, D. C.; Luzyanin, K. V.; Higgins, S. J.; Nichols, R. J.; Beeby, A.; Low, P. J.; Li, B.; Niu, L., Single-Molecule Conductance of ViologenCucurbit[8]uril Host-Guest Complexes. ACS Nano 2016, 10 (5), 5212-20.

9. Rao, S. S.; Lande, D. N.; Gejji, S. P., Density functional theory investigations on binding and spectral features of complexes of ferrocenyl derivatives with cucurbit [7] uril. J. Mol. Liq. 2016, 216, 298-308.

10. Pinjari, R. V.; Gejji, S. P., Electronic structure, molecular electrostatic potential, and NMR chemical shifts in cucurbit [n] urils ( $n=5-8)$, ferrocene, and their complexes. J. Phys. Chem. A 2008, 112 (49), 12679-12686.

11. Kanthasamy, K.; Ring, M.; Nettelroth, D.; Tegenkamp, C.; Butenschon, H.; Pauly, F.; Pfnur, H., Charge Transport through Ferrocene 1,1'-Diamine Single-Molecule Junctions. Small 2016, 12 (35), 4849-4856.

12. Kim, J.; Jung, I.-S.; Kim, S.-Y.; Lee, E.; Kang, J.-K.; Sakamoto, S.; Yamaguchi, K.; Kim, K., New cucurbituril homologues: syntheses, isolation, characterization, and X-ray crystal structures of cucurbit [n] uril ( $n=5,7$, and 8). J. Am. Chem. Soc. 2000, 122 (3), 540-541.

13. Pemberton, B. C.; Singh, R. K.; Johnson, A. C.; Jockusch, S.; Da Silva, J. P.; Ugrinov, A.; Turro, N. J.; Srivastava, D.; Sivaguru, J., Supramolecular photocatalysis: insights into cucurbit [8] uril catalyzed photodimerization of 6-methylcoumarin. Chem. Commun. 2011, 47 (22), 6323-6325.

14. Walker, S.; Oun, R.; Mclnnes, F. J.; Wheate, N. J., The potential of cucurbit [n] urils in drug delivery. Isr. J. Chem. 2011, 51 (5 - 6), 616-624.

15. Hosenbocus, S.; Chahal, R., Amantadine: a review of use in child and adolescent psychiatry. $J$. Can.Acad.Child Adolesc. Psychiatry 2013, 22 (1), 55.

16. Park, Y. S.; Whalley, A. C.; Kamenetska, M.; Steigerwald, M. L.; Hybertsen, M. S.; Nuckolls, C.; Venkataraman, L., Contact chemistry and single-molecule conductance: a comparison of phosphines, methyl sulfides, and amines. J. Am. Chem. Soc. 2007, 129 (51), 15768-15769.

17. Hybertsen, M. S.; Venkataraman, L.; Klare, J. E.; Whalley, A. C.; Steigerwald, M. L.; Nuckolls, C., Amine-linked single-molecule circuits: systematic trends across molecular families. J. Phys.: Condens. Matter 2008, 20 (37), 374115.

18. Quek, S. Y.; Kamenetska, M.; Steigerwald, M. L.; Choi, H. J.; Louie, S. G.; Hybertsen, M. S.; Neaton, J.; Venkataraman, L., Mechanically controlled binary conductance switching of a singlemolecule junction. Nat. Nano. 2009, 4 (4), 230. 